\title{
Incidence and prognosis of undifferentiated cancers of the digestive system: a population-based cohort study
}

\author{
Jingping Qiu ${ }^{1,2}$, Yan Xin ${ }^{2}$ \\ ${ }^{1}$ Department of Radiation oncology, The First Hospital of China Medical University, Shenyang, China; ${ }^{2}$ Laboratory of Gastrointestinal Onco- \\ Pathology, Cancer Institute and General Surgery Institute, The First Hospital of China Medical University, Shenyang, China \\ Contributions: (I) Conception and design: All authors; (II) Administrative support: Y Xin; (III) Provision of study materials or patients: All authors; \\ (IV) Collection and assembly of data: J Qiu; (V) Data analysis and interpretation: All authors; (VI) Manuscript writing: J Qiu; (VII) Final approval of \\ manuscript: All authors. \\ Correspondence to: Yan Xin, MD, PhD. Laboratory of Gastrointestinal Onco-Pathology, Cancer Institute and General Surgery Institute, The First \\ Hospital of China Medical University, 155 Nanjing North Street, Heping District, Shenyang 110001, China. Email: yxin@cmu.edu.cn.
}

Background: Cases of undifferentiated cancers of the digestive system (UCDS) are occasionally encountered in clinical practice; however, no large-scale studies have described their characteristics. This study aimed to investigate the incidence, prognosis, and treatment strategies of UCDS.

Methods: We used the data from the Surveillance, Epidemiology, and End Results database to evaluate clinical characteristics in UCDS patients. A cohort of 1,372 patients was included to estimate UCDS incidence; another cohort of 1,816 patients was followed up until December 31, 2017 to evaluate treatment and prognosis. The incidence, trends, treatments, overall survival, and cause-specific survival were assessed.

Results: The estimated incidence of UCDS was 0.136 per 100,000 people-years [ $95 \%$ confidence interval (CI): 0.129-0.143] from 1975 to 2016 with respect to sex, age, year of diagnosis, race, region, site, income, and education. The age-adjusted rates of UCDS dropped from 0.567 per 100,000 people-years in 1978 to 0.031 per 100,000 people-years in 2016 . The rates of surgery, beam radiation, and chemotherapy in patients with UCDS were $37.3 \%, 17.3 \%$, and $30.7 \%$, respectively. Overall, the 5-year cause-specific survival rate was $18 \%$, with a median cause-specific survival of 4 months. Patient prognosis improved significantly over the decades; stage, site, year of diagnosis, surgery, beam radiation, and chemotherapy were independent prognostic factors for UCDS mortality.

Conclusions: The incidence of UCDS has decreased in the United States over the past 40 years. Patients benefited from surgery, beam radiation, and chemotherapy. The survival of patients with UCDS has improved. Further research on developing decision-making recommendations for UCDS treatment is crucial.

Keywords: Undifferentiated cancer of the digestive system (UCDS); incidence; prognosis; treatment

Submitted Feb 15, 2020. Accepted for publication Oct 10, 2020.

doi: 10.21037/atm-20-1615

View this article at: http://dx.doi.org/10.21037/atm-20-1615

\section{Introduction}

Cancers of the digestive system have the highest prevalence and mortality, accounting for 328,030 cases and 165,460 deaths in the United States (US) in 2019 (1). Among these cancers, adenocarcinoma is the most common histological type. Differentiation is an indicator of malignancy and a predictor of survival and treatment resistance (2). However, the characteristics of undifferentiated cancers of the digestive system (UCDS) have not been fully elucidated. A deeper knowledge of UCDS will help improve community health by developing public health prevention strategies, formulating health insurance policies, and identifying multidisciplinary treatments.

Available publications on UCDS are scarce; and thus, this 
disease is not well understood. In terms of epidemiology, the most common site of UCDS is the stomach, as reported by researchers in East Asia who observed it during endoscopic resection for early gastric cancer $(3,4)$. Other sites such as the pancreas and hepatobiliary organs have also been reported (5-8). However, reports on the incidence of UCDS are limited. Moreover, the time trends of UCDS incidence have not been analyzed. For the treatment of undifferentiated early gastric cancer, endoscopic and surgical resections were shown to be effective $(3,9,10)$. However, for regional and metastatic undifferentiated gastric cancer and UCDS at other sites, multidisciplinary treatment strategies were used without a specified value assigned for each treatment $(7,11,12)$.

Therefore, it is important to assess the malignant potential of UCDS. We put forth the following hypotheses: (I) UCDS incidence has decreased over time and the incidence is associated with demographic and socioeconomic factors; (II) patients with UCDS may benefit from systemic therapies such as chemotherapy rather than from regional treatments such as surgery and radiotherapy; and (III) the prognosis is poor even for early-stage disease and is independent of the primary tumor site, but it may have improved in recent decades. We excluded oral tumors in this study because squamous cell carcinoma is the most common histological type in these tumors; therefore, oral cancer was classified as squamous cell carcinoma of the head and neck.

Given the low frequency of UCDS, the best way to evaluate its epidemiology is through the use of large population-based registries containing longitudinal data. The Surveillance, Epidemiology, and End Results (SEER) database is a comprehensive source of information from the National Cancer Institute, which has provided cancer incidence and population data annually since 1973. Thus, this cohort analysis aimed to improve our knowledge of UCDS, which is important from both clinical and public health perspectives. Therefore, we comprehensively evaluated UCDS incidence, time trends, treatments, and outcomes using the data from the SEER database. We present the following article in accordance with the STROBE reporting checklist (available at http://dx.doi. org/10.21037/atm-20-1615).

\section{Methods}

\section{Data sources}

Data collection for the SEER database began in 1973 using various locations and sources throughout the US. The following data are collected continuously: incidence; patient demographics; tumor site, morphology, and stage; treatment; and follow-up. The SEER database contains enriched patient data in terms of detail and longitudinal information from expanding registries. The Surveillance Research Program of the National Cancer Institute's Division of Cancer Control and Population Sciences provides analytical tools and methodological support required for collecting, analyzing, interpreting, and disseminating population-based statistics. We obtained data corresponding to 1975-2016 from the SEER Program (www.seer.cancer.gov) SEER*Stat Database, submitted in November 2018 and released in April 2019. The latest released SEER data cover approximately $34.6 \%$ of the US population (13).

To maximize the use of information, two cohorts were created. One cohort, used to estimate incidence and time trends, was created from the incidence database of the SEER 9 registry (Atlanta, Connecticut, Detroit, Hawaii, Iowa, New Mexico, San Francisco-Oakland, Seattle-Puget Sound, and Utah). The other cohort, used to analyze treatment strategies and survival, was created from a case listing of the SEER 18 registry (SEER 9, plus Los Angeles, San Jose-Monterey, rural Georgia, Alaska Native Tumor Registry, Greater California, Greater Georgia, Kentucky, Louisiana, and New Jersey), covering approximately $28 \%$ of the US population (http://seer.cancer.gov). The study conformed to the provisions of the Declaration of Helsinki (as revised in 2013). This study was deemed to be exempt from full institutional review board review by China Medical University due to its retrospective nature.

\section{Patient selection}

UCDS cases were identified on the basis of (I) International Classification of Diseases for Oncology, Third Edition (ICD-O-3) codes for primary sites in the digestive system with the following ones included: the esophagus (C150C159); stomach (C160-C169); small intestine (C170C179); cecum (C180); appendix (C181); ascending colon (C182); hepatic flexure (C183); transverse colon (C184); splenic flexure (C185); descending colon (C186); sigmoid colon (C187); large intestine, not otherwise specified (NOS) (C188-C189, C260); rectosigmoid junction (C199); rectum (C209); anus, anal canal, and anorectum (C210-C212, C218); liver (C220); intrahepatic bile duct (C221); gallbladder (C239); other biliary tract sites (C240- 
C249); pancreas (C250-C259); retroperitoneum (C480); peritoneum, omentum, and mesentery (C481-C482); and other digestive organs (C268-C269, C488) and (II) undifferentiated histologic type (ICD-O-3 histology codes 8020/3: Carcinoma, undifferentiated, NOS).

\section{Information extraction}

We extracted data on the following variables: sex, age at diagnosis, year of diagnosis, race, marital status, region, tumor site, and tumor stage. Information on regions was recorded based on Contract Health Service Delivery Area counties, including those in the Northern Plains, Southwest region, East region, and Pacific Coast. Stage information was extracted from "SEER historic stage A", which was a simplified version of stage: in situ, localized, regional, distant, and unknown. We chose three variables, namely income, education, and employment, to indicate the socioeconomic status. Furthermore, the socioeconomic status was determined depending on the county of residence in each case and estimated from the Census 2012-2016 American Community Survey. For socioeconomic status, the patients were stratified into two categories according to the median level. Surgery information was extracted based on the SEER variable of "Reason no cancer directed surgery". The status of patients at the time of the last follow-up was evaluated based on the SEER variable of "cause of death (COD) to site recode". We used the variable "survival time months" to extract data on the time from the date of diagnosis to the date of the last follow-up. The follow-up cutoff date in this study was December 31, 2016.

\section{Statistical analysis}

Incidence was analyzed using SEER*Stat, version 8.3.6 (Surveillance Research Program, National Cancer Institute, seer.cancer.gov/seerstat). Trends in incidence and differences between trends were analyzed using Joinpoint Regression Program 4.7.0.0 (Statistical Methodology and Applications Branch, Surveillance Research Program, National Cancer Institute) with the joinpoint regression model and permutation tests for identifying changes in trends (14). Statistical analysis was performed using IBM SPSS Statistics for Windows, version 24.0 (IBM Corp., Armonk, NY, USA). Graphs were created in GraphPad Prism version 8.0.0 (GraphPad Software, Inc.).

The incidence rates per 100,000 people-years were age adjusted to the 2000 US standard population. Percent changes were calculated at intervals of 1 year for each endpoint. The rates of treatment were compared between groups using chisquare tests. The 1-, 3-, and 5-year cause-specific survival (CSS) rates were estimated using the life table method; median survival rates were estimated using Kaplan-Meier analyses and compared using log-rank tests. Prognostic factors were identified by univariate regression analysis, wherein hazard ratios (HRs) with 95\% confidence intervals (CIs) were calculated to estimate the mortality risk. A multivariable analysis was further performed to identify independent prognostic factors using a Cox proportional hazard model. A P value $<0.05$ was used to indicate statistical significance.

The independent prognostic factors derived from the Cox multivariate analysis were selected to construct a nomogram of CSS. To improve the application value of the data, only patients diagnosed between 1997 and 2016 were included, and the variable of region was eliminated. The nomogram was constructed using $\mathrm{R}$ software, version 3.6.1 (R Foundation for Statistical Computing) and Rstudio, version 1.2.1335 (RStudio, Inc.). The bootstrap method (sampling was repeated 1,000 times) was used for internal verification, and the conformity of the model was evaluated using the C-index and a calibration diagram.

\section{Results}

\section{Incidence}

Between 1975 and 2016, a total of 1,372 patients with UCDS were identified in the SEER 9 registry. The overall incidence of UCDS during that period was 0.136 per 100,000 people-years (95\% CI: 0.129-0.143). The incidence was dependent on sex, age, year of diagnosis, race, region, site, income level, and education level (Table 1). The incidence was higher in male and black populations; for the incidence, the ratio of women to men was 0.604 and that of black to white people was 1.456 . More than half of the patients were diagnosed before 1986, and the proportion of UCDS cases has been decreasing gradually (Figure 1A). Older age was associated with UCDS tumorigenesis, a pattern consistent with that in digestive cancer (Figure 1B). Few patients were under 50 years of age, and the incidence among patients aged $\geq 70$ years was three times higher than that among patients aged 50-70 years. Among geographical regions, higher incidences were observed in the Northern Plains and Pacific Coast, while the lowest incidence was observed in the Southwest region. Advanced-stage disease was predominant, and patients with regional and distant 
Table 1 Frequency, incidence and rate ratios of the undifferentiated cancers in the digestive system

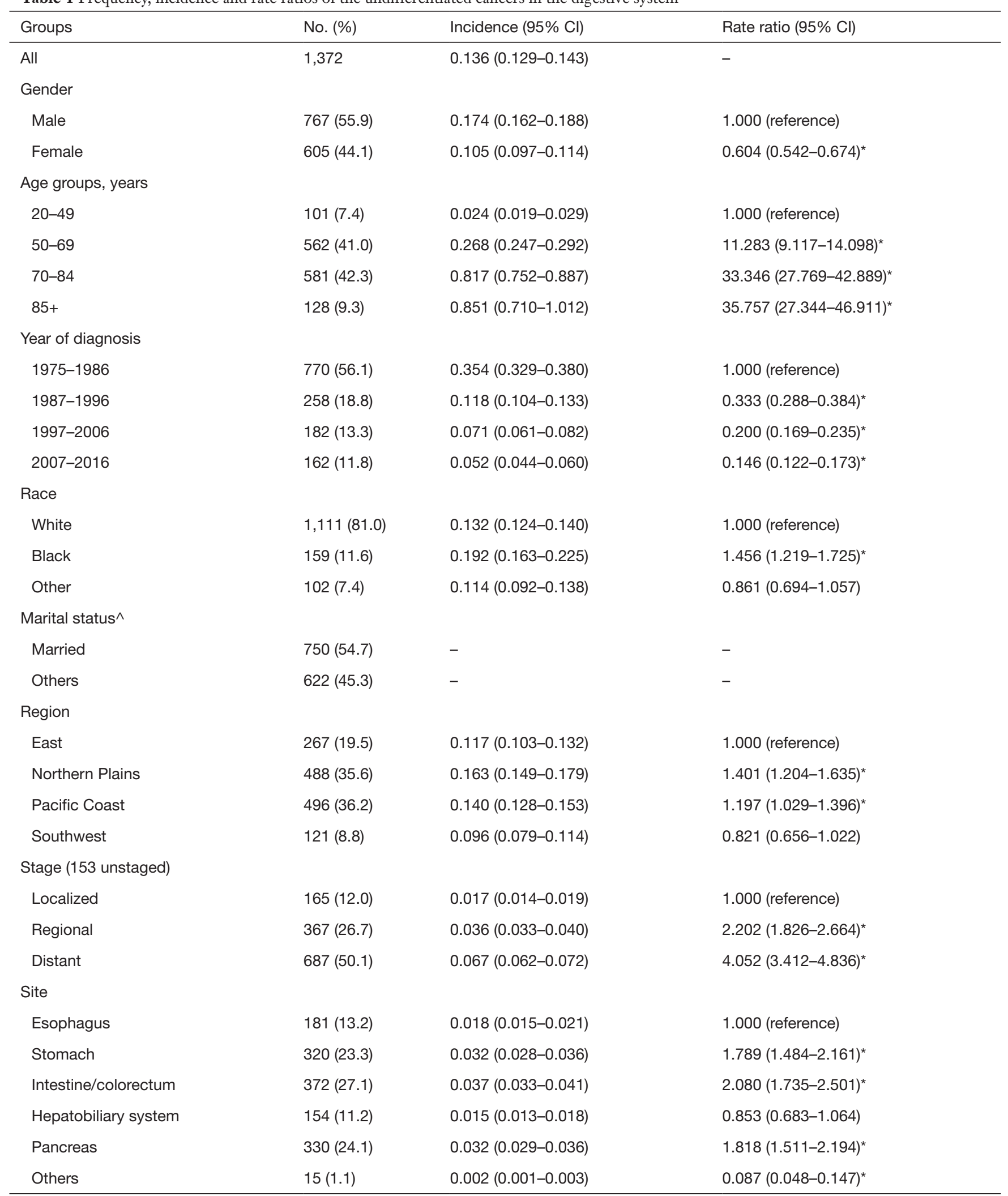

Table 1 (continued) 
Table 1 (continued)

\begin{tabular}{|c|c|c|c|}
\hline Groups & No. (\%) & Incidence (95\% Cl) & Rate ratio $(95 \% \mathrm{Cl})$ \\
\hline \multicolumn{4}{|l|}{ Income } \\
\hline Low level & $363(26.5)$ & $0.160(0.144-0.177)$ & 1.000 (reference) \\
\hline High level & $1,009(73.5)$ & $0.129(0.121-0.137)$ & $0.806(0.713-0.912)^{*}$ \\
\hline \multicolumn{4}{|l|}{ Education } \\
\hline \multicolumn{4}{|l|}{ Employment } \\
\hline Low level & $749(54.6)$ & $0.139(0.129-0.149)$ & 1.000 (reference) \\
\hline High level & $623(45.4)$ & $0.132(0.122-0.143)$ & $0.949(0.852-1.058)$ \\
\hline
\end{tabular}

Incidence are per 100,000 and age-adjusted to the 2000 US standard population. * $\mathrm{P}<0.05 ; \wedge$, marital status as diagnosis is not a population variable and cannot be used when calculating rates, therefore the table contains frequency only.
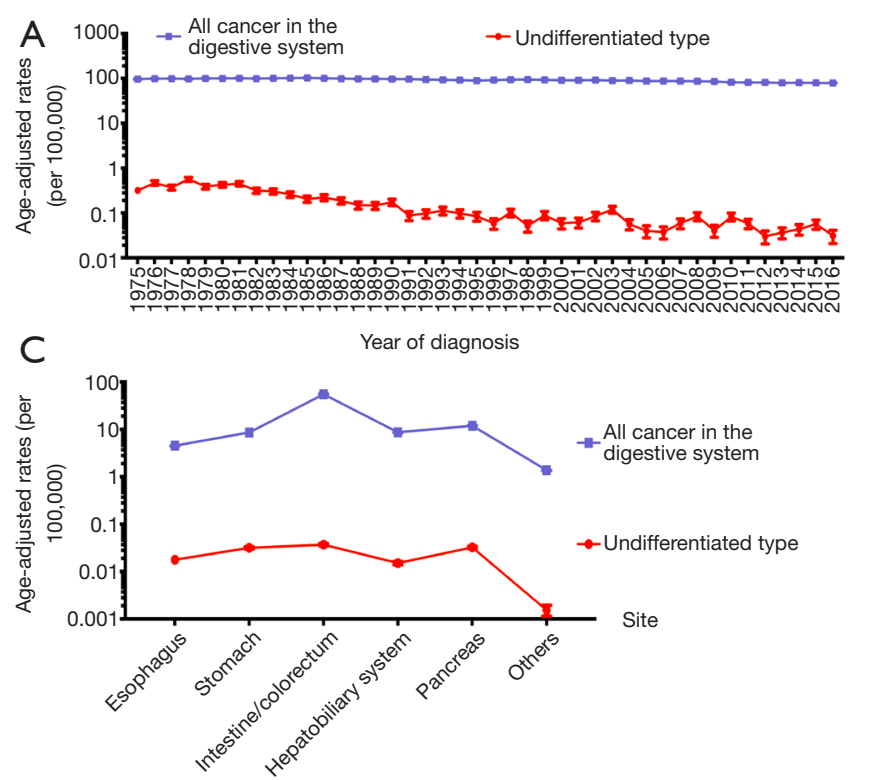
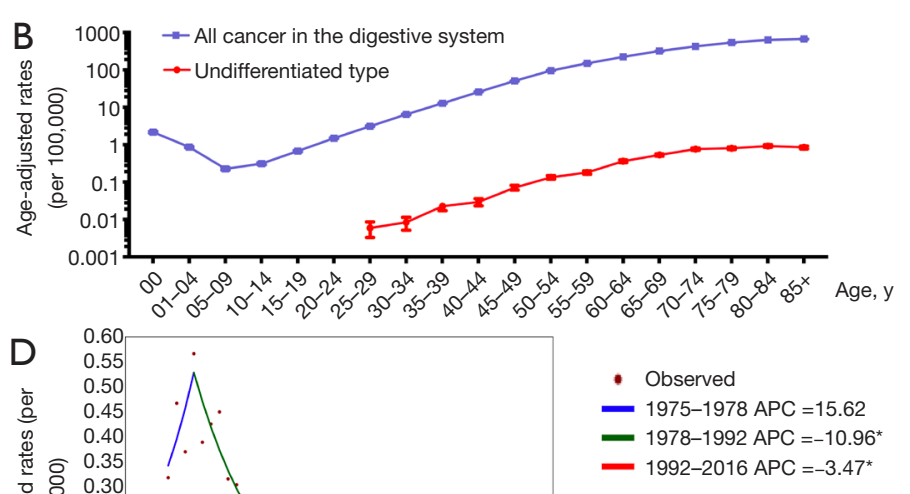

Figure 1 Age-adjusted rates and trends of undifferentiated cancers of the digestive system. The age-adjusted rates of cancers in the digestive system and those of undifferentiated cancers of the digestive system (UCDS) according to the year of diagnosis (A), age (B), and anatomical site (C). (D) The trends of age-adjusted rates of UCDS from 1975 to 2016.

metastases accounted for $76.8 \%$ of cases. The incidences were high for UCDS in the stomach, intestines/colorectum, and pancreas, similar to the pattern observed in digestive cancer (Figure 1C). Higher incidence was associated with lower levels of income and education.

\section{Trends}

The age-adjusted rates of UCDS ranged from 0.318 to 0.567 per 100,000 people-years between 1975 and 1978, but this difference was not significant $(\mathrm{P}=0.2)$; then, the rate rapidly dropped to 0.097 in 1992 , with an annual percentage change of -11.0 (95\% CI: -13.5 to $-8.3, \mathrm{P}<0.05)$. The value slowly declined to 0.031 in 2016, with an annual percentage change of -3.5 (95\% CI: -5.2 to $-1.7, \mathrm{P}<0.05$ ) (Figure $1 D$ ). Stratification of the cohort by sex or race to conduct pairwise comparisons showed that the trends observed were independent of sex and race. 


\section{Treatment}

In total, 1,816 patients with UCDS qualified for the study. The rates of surgery, beam radiation, and chemotherapy were $37.3 \%, 17.3 \%$, and $30.7 \%$, respectively. Although the treatment of UCDS was multimodal, the choice of treatment modality depended on sex, age, year of diagnosis, marital status, region, site, stage, and multiple primary malignant tumors (Table S1). Male patients and those with localized/regional-stage disease were more likely to undergo surgery, radiation therapy, and chemotherapy. Although the rates of radiotherapy or chemotherapy were low in patients over 85 years of age, the rate of surgery in these patients was higher than that in younger patients. Over the decades, more patients underwent surgery and chemotherapy and fewer received radiation therapy. The rate of radiation in patients in the Southwest region was three-fold lower than that in those in other regions. Patients with colorectal cancer had the highest rate of surgery, while those with esophageal cancer had the highest rate of radiation, similar to the rates for tumors in the same anatomic site.

\section{Survival}

Overall, 1,795 patients were followed up, corresponding to a follow-up rate of $98.8 \%$. The 5 -year CSS rate of UCDS was $18 \%$, with a median CSS of 4 months. Multivariable analysis showed that stage, site, year of diagnosis, surgery, beam radiation, and chemotherapy were independent prognostic factors of UCDS mortality (Figure 2 and Table 2). Advanced stage was associated with a worse prognosis. The median survival was nearly 4 years for localized stage disease compared with 9 and 2 months for regional stage disease and distant stage disease, respectively. Patients benefited from each of the three treatment modalities, with surgery extending survival from 3 to 15 months. Prognosis has improved significantly over the decades, with the 5-year CSS rate increasing to $28 \%$ in the last 20 years. Regarding tumor site, colorectal UCDS had the best prognosis. Although multiple primary malignant tumors were significantly related to prognosis, they could be considered an independent prognostic factor due to chronological bias.

\section{Nomogram}

We included the factors of site, stage, surgery, beam radiation, and chemotherapy in a nomogram to predict the 3-, 5-, and 10-year CSS rates (Figure 3A). We used the bootstrap method for internal verification and found a C-index of 0.762 . The prediction calibration curve fitted well with the standard curve in the calibration diagram, indicating favorable accuracy of the prediction model (Figures 3B,C,D).

\section{Discussion}

In this study, we found that (I) the incidence of UCDS was 0.136 per 100,000 people-years from 1975 to 2016, and it decreased to 0.031 per 100,000 people-years in 2016 ; (II) the management of UCDS was multidisciplinary: the rates of surgery, beam radiation, and chemotherapy were $37.3 \%$, $17.3 \%$, and $30.7 \%$, respectively, and patients benefited from each of the treatment modalities, especially surgery; (III) the prognosis of UCDS was poor, with a 5 -year CSS rate of $18 \%$; and (IV) the independent prognostic factors were stage, site, year of diagnosis, surgery, beam radiation, and chemotherapy.

We confirmed the hypothesis of a decreasing incidence of UCDS. The observed incidence was comparable to that reported previously. The Worldwide Esophageal Cancer Collaboration has indicated that undifferentiated carcinomas constituted $0.20 \%(26 / 13,300)$ of esophageal cancers using data from 33 institutions from six continents, mainly from Asia (15). Immunohistochemistry is crucial for the pathological diagnosis of UCDS. The histopathologic subtypes of digestive cancer have become well recognized owing to the widespread application of immunohistochemistry in recent decades $(16,17)$. Our findings suggested a gradually decreasing incidence of UCDS. This consistency in timelines indicated that the popularity of immunohistochemistry could be the cause of the declining incidence of UCDS.

We also reported the demographic factors associated with UCDS incidence by analyzing a large number of cases. Previously, Singhi et al. evaluated 16 cases of resected undifferentiated esophageal carcinomas and reported that the patients were mostly elderly and predominantly male (18). Park et al. conducted a study on a group of patients with early gastric cancer who underwent curative endoscopic submucosal dissection; $7.2 \%(113 / 1,560)$ of the patients had undifferentiated-type cancer, which had an association with male sex (19). Zhang et al. reported a higher incidence of undifferentiated carcinoma of the pancreas in men and in those with a median age of 60 years (20). However, a study on undifferentiated carcinoma of the gallbladder reported female predominance (11). Furthermore, Brozek et al. 

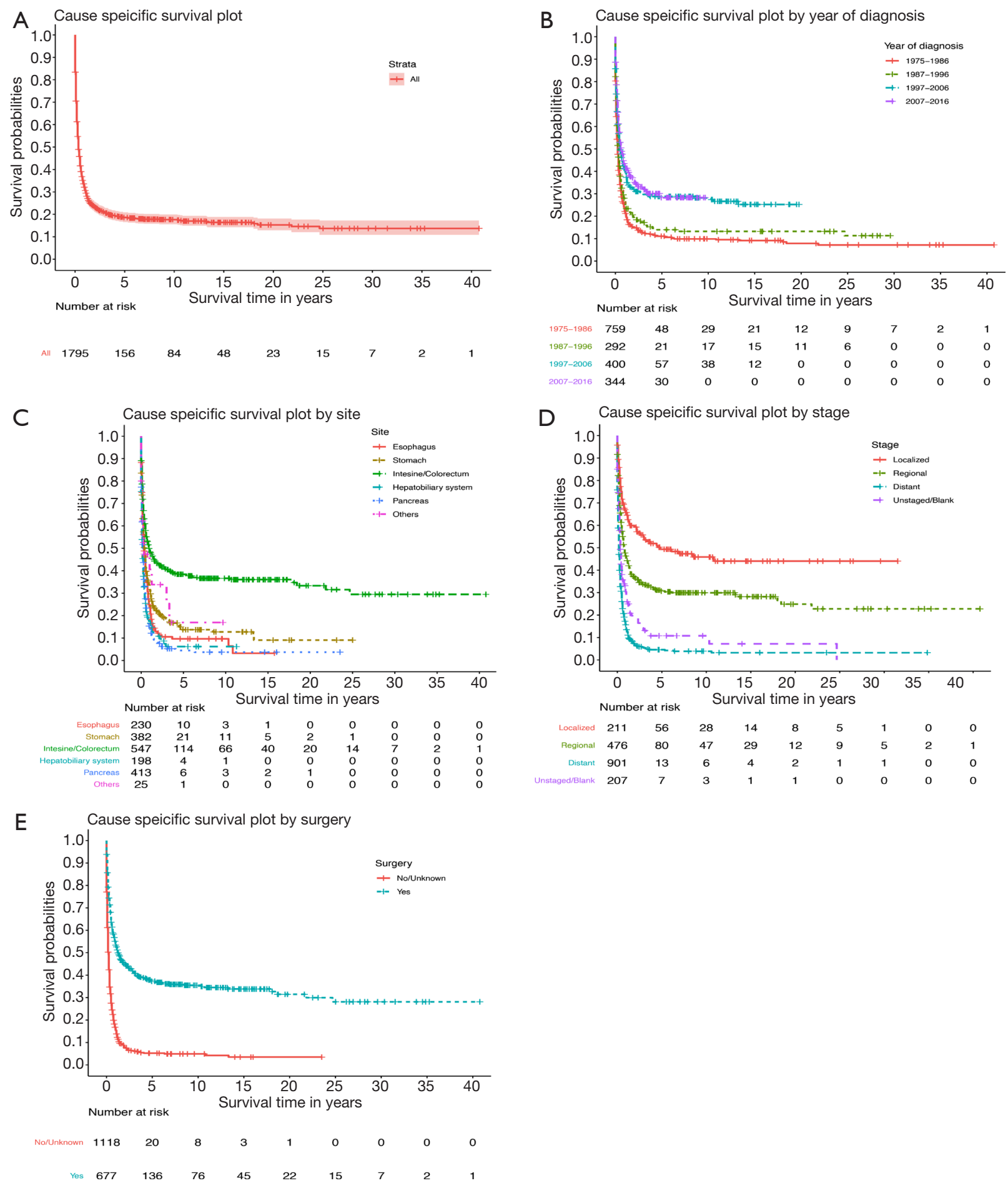

Figure 2 Cause-specific survival. The cause-specific survival plots of all patients (A) stratified by the year of diagnosis (B), site (C), stage (D), and surgery $(\mathrm{E})$. 
Table 2 Univariate and multivariate survival analyses for cause specific survival of undifferentiated cancers in digestive system

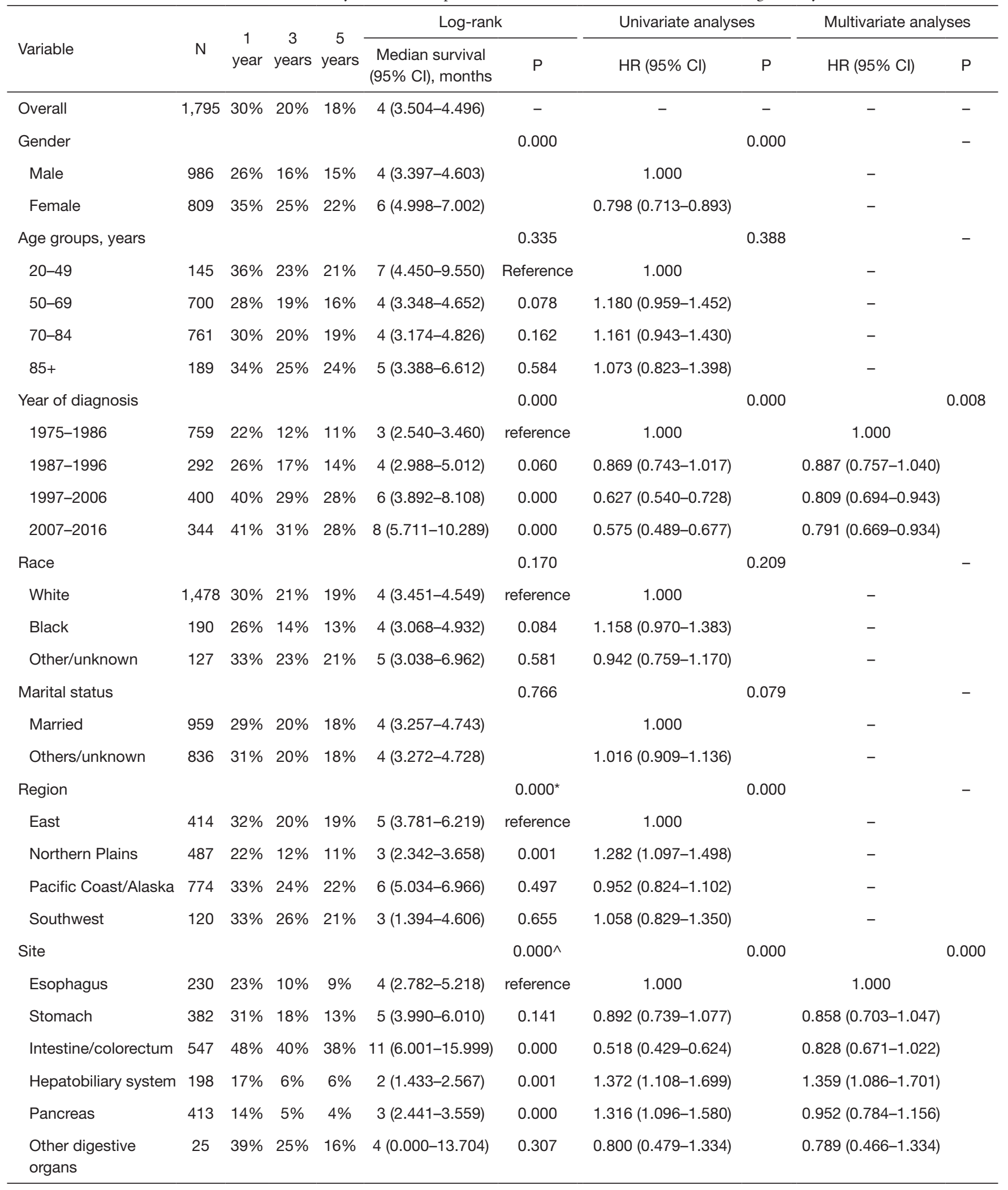

Table 2 (continued) 
Table 2 (continued)

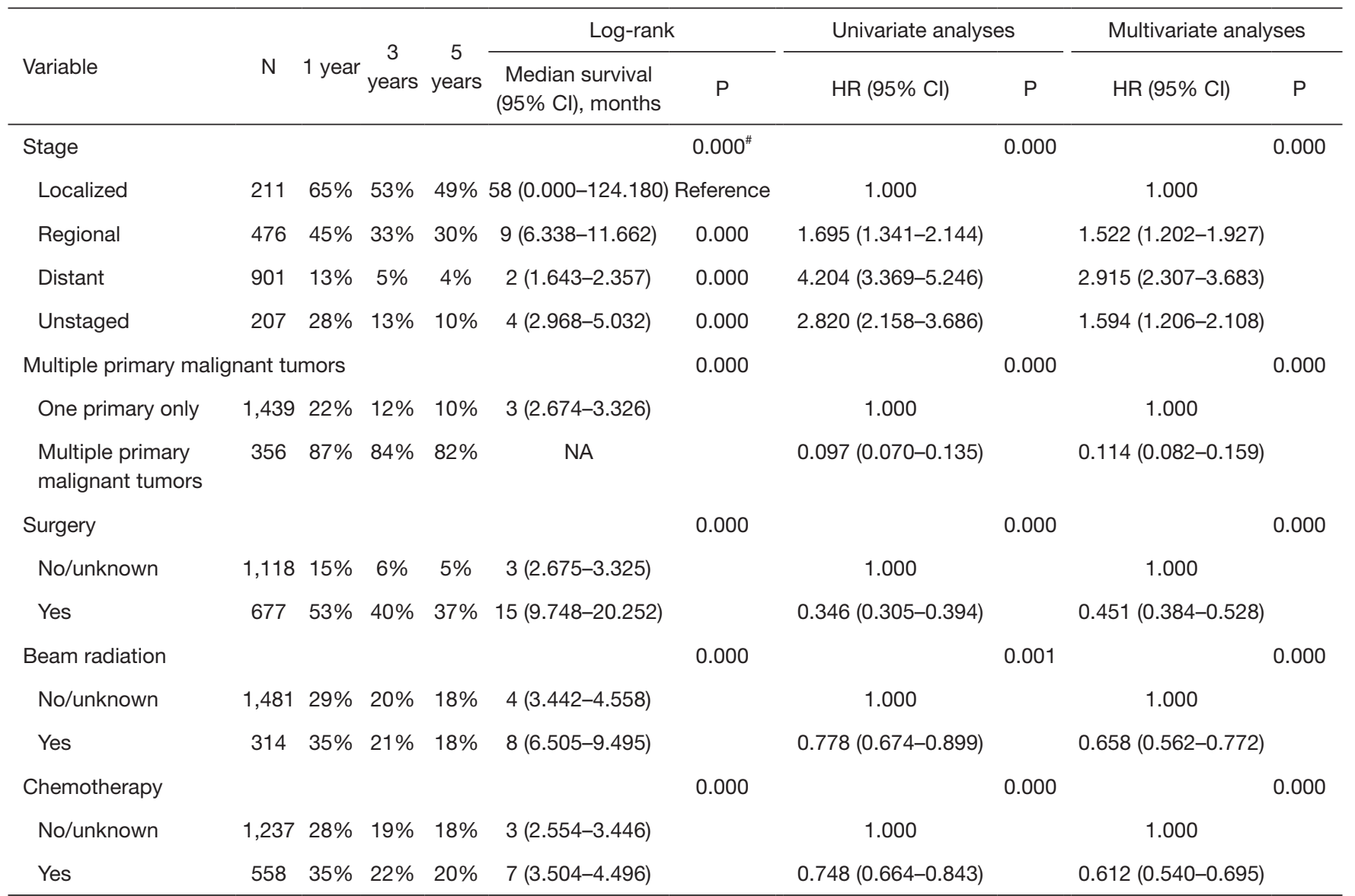

*, Northern Plains vs. Pacific Coast/Alaska, $\mathrm{P}=0.000$; Northern Plains vs. Southwest, $\mathrm{P}=0.095$; Pacific Coast/Alaska vs. Southwest, $\mathrm{P}=0.339$; $\wedge$, stomach vs. intestine/colorectum, $\mathrm{P}=0.000$; vs. hepatobiliary system, $\mathrm{P}=0.000$; vs. pancreas, $\mathrm{P}=0.000$; intestine/colorectum vs. hepatobiliary system, $\mathrm{P}=0.000$; vs. pancreas, $\mathrm{P}=0.000$; hepatobiliary system vs. pancreas, $\mathrm{P}=0.674$; \#, regional vs. distant, $\mathrm{P}=0.000$; CSS, cause specific survival; $\mathrm{HR}$, hazard ratio; $\mathrm{Cl}$, confidence interval.

observed that despite the higher incidence in older women than in older men, the incidences of poorly differentiated and undifferentiated rectal cancer at an early age were similar between women and men. The authors attributed this difference to the lower vitamin $\mathrm{D}$, calcium, and estrogen levels in women of advanced age and reported that adequate vitamin $\mathrm{D}$ and calcium intakes protected premenopausal women against more aggressive cancers (21). Notably, the proportion of undifferentiated cancer cases was high in some Asian studies. For example, in a study in Japan, Kakinoki et al. reported that $30.1 \%(116 / 386)$ of gastric cancers were of the undifferentiated type (22). Furthermore, Kwon et al. reported that undifferentiated carcinoma accounted for $21.9 \%$ and $48.8 \%$ of early and advanced gastric cancers, respectively, in a Korean study (23). Such high proportions are inconsistent with the results of this study and our clinical experience, suggesting that there are differences in prevalence or diagnostic criteria among these countries (24).

Our study showed that low socioeconomic status was associated with a high incidence of UCDS. Previous studies have reported inconsistent results regarding the correlation between socioeconomic status and digestive cancer incidence. First, many researchers reported a positive correlation between socioeconomic status and the incidence of digestive cancers. In France, a study of 189,144 cancer cases reported that a low socioeconomic status was associated with a high incidence of stomach cancer and liver cancer in both men and women; it was also associated with the incidence of esophageal cancer and pancreatic cancer in men (25). In the US, studies have reported that 


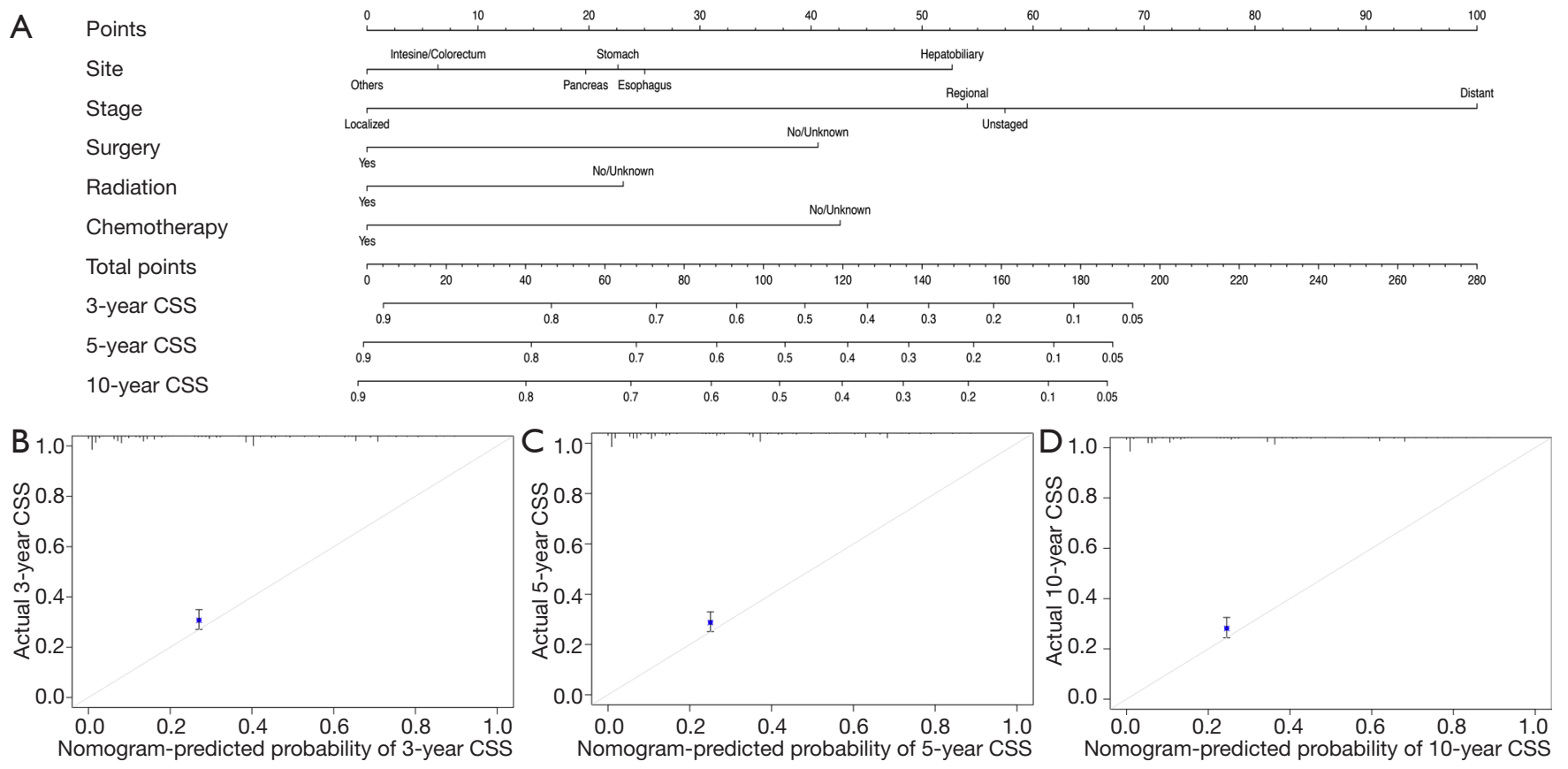

Figure 3 Nomogram of UCDS. The nomogram of UCDS with a 100-point scale (A) and nomogram-predicted probabilities of 3-, 5-, and 10-year cause-specific survival, respectively (B,C,D). UCDS, undifferentiated cancers of the digestive system; CSS, cause-specific survival.

the incidences of stomach, colorectum, and liver cancers were higher in a population with low levels of income and education (26,27). A Finnish study found that colon cancer incidence was higher in highly educated people than in those with a basic education (28). An American study of 40,314 patients with colorectal cancer found a negative correlation between socioeconomic status and colorectal cancer incidence (29). In contrast, a Swedish study including 82,686 patients with colorectal cancer reported that disposable income and education levels were not related to the incidence of colorectal cancer (30). This inconsistency in correlation between UCDS incidence and socioeconomic status indicates the heterogeneity in tumorigenesis of undifferentiated cancers; furthermore, the differences in mortality and morbidity may reflect differences in smoking status, obesity, lack of exercise, diet, alcohol consumption, screening, and treatment (27). Therefore, our results are significant from both public health and clinical perspectives. In terms of prevention strategies, our research identified high-risk populations for screening. Prevention strategies for cancers should be applied to all individuals, regardless of their socioeconomic status. However, screening of patients with low income and education levels may reduce the heterogeneity in UCDS incidence. Similarly, reasonable allocation of medical budgets should be considered alongside health insurance policies. In terms of tumorigenesis of UCDS, the high incidence in people with low income and education levels is a clue for identifying the pathogenesis. Potentially, preventive measures directed at changing certain lifestyle choices may narrow down health differences based on income and education levels.

Treatment choices differed in terms of sex, age, year of diagnosis, marital status, region, tumor site, and stage. Although patients benefited from surgery, radiotherapy, and chemotherapy, the rate of radiotherapy has dropped in recent decades. Owing to the relative rarity of UCDS and the lack of clinical trials, therapeutic guidelines or recommendations are not available (31). Literature reports have suggested a variety of regional and systemic treatment strategies; however, complete response and long-time survival were achieved in only a few cases $(32,33)$. Reis Neto et al. studied 287 patients with rectal adenocarcinoma treated with radiotherapy followed by surgery and reported that preoperative radiotherapy decreased the proportion of undifferentiated cells, indicating that undifferentiated rectal cancer cells were more sensitive to the radiation than differentiated cancer cells (34). The effectiveness of chemotherapy and combined regimens for undifferentiated 
carcinoma remains unclear because of the small number of reported cases (12). The present large populationbased cohort study provided a higher level of evidence for treatment.

In this study, surgery has increased the survival in UCDS patients by more than 1 year and it was the most effective treatment among the three traditional treatments available, indicating that surgery should be the first-line treatment in operative cases, especially for colorectal UCDS. This indicated that invasive and metastatic events were not always inevitable in regional cases. Similarly, several studies have revealed lymph node metastasis rates of $0-1.1 \%$ after curative resection of undifferentiated gastric carcinoma; the authors suggested that endoscopic submucosal dissection should be considered a possible treatment for undifferentiated early gastric cancer and that undifferentiated cancers do not require a more thorough surgical resection and a wider range of lymph node dissection than other pathologic types of cancer $(11,19,35)$. However, other studies have suggested that the risk of lymph node metastasis in pancreatic undifferentiated cancer was not higher than that in pancreatic adenocarcinoma $(20,36,37)$.

A unique finding of this study was the identification of the independent prognostic factors of UCDS. Although the prognosis of UCDS is poor, it has improved over the past 40 years, with the 5 -year survival rate of local-stage patients reaching $49 \%$. Previously, the prognosis was estimated using small sample sizes. For example, several studies have reported poor prognosis in hepatobiliary cancer, with a median survival as short as 3 months $(7,8,11,12)$. A study on eight cases of undifferentiated carcinoma of the gallbladder reported a median survival of 7.3 months and a prognostic advantage for early-stage disease (7). A Japanese study reported that tumor size $>10 \mathrm{~cm}$ was an independent prognostic factor in patients with advanced undifferentiated gastric carcinoma and found that the undifferentiated type was more invasive, with a higher incidence of peritoneal dissemination, while the differentiated type was more likely to metastasize to the liver (38). A Korean study including 4,236 patients with early undifferentiated-type gastric cancer reported an increased rate of lymph node metastasis with increased $\mathrm{T}$ stage and favorable overall survival in younger patients. Notably, the overall survival rates were $95.5 \%$ and $86.1 \%$ in the old and young groups, respectively; this may be due to the histological diversity in this study: poorly differentiated adenocarcinoma, signet-ring cell carcinoma, and mucinous carcinoma were categorized as undifferentiated-type tumors (39).
Given the large number of cases and the long durations of observation and follow-up, the present study provided a comprehensive epidemiologic picture of UCDS. Determination of the incidence of UCDS provides a scientific basis for the development of hygiene policies. Furthermore, the prognostic factors identified are critical for clinical decision-making. The developed nomogram can be used by clinicians to evaluate UCDS mortality.

Our study had several limitations. First, central verification of the pathologic diagnosis was lacking, and some specific pathological subtypes could not be further analyzed. Second, data on performance status and systemic treatment agents were not available. Finally, the inherent limitations of retrospective, population-based, cohort studies include selection, information, and confounder biases. These limitations may raise concerns about the generalizability of these findings. Therefore, prospective studies and randomized trials are required to verify these findings.

\section{Conclusions}

The incidence of UCDS yields lower in the US in the past 40 years. The survival of patients with UCDS has improved. Stage, site, year of diagnosis, surgery, beam radiation, and chemotherapy were independent prognostic factors for UCDS.

\section{Acknowledgments}

We would like to thank Editage (www.editage.cn) for English language editing.

Funding: This study was supported by the National Natural Science Foundation of China (No. 81971584 and No. 81071650); Special Foundation for Science and Technology Program in Liaoning Province, China (2013225585); and Supporting Project for Climbing Scholars in Liaoning Provincial Universities, China (2009).

\section{Footnote}

Reporting Checklist: The authors have completed the STROBE reporting checklist. Available at http://dx.doi. org/10.21037/atm-20-1615

Peer Review File: Available at http://dx.doi.org/10.21037/ atm-20-1615

Conflicts of Interest: Both authors have completed the 
ICMJE uniform disclosure form (available at http://dx.doi. org/10.21037/atm-20-1615). The authors have no conflicts of interest to declare.

Ethical Statement: The authors are accountable for all aspects of the work in ensuring that questions related to the accuracy or integrity of any part of the work are appropriately investigated and resolved. The study conformed to the provisions of the Declaration of Helsinki (as revised in 2013). This study was deemed to be exempt from full institutional review board review by China Medical University due to its retrospective nature.

Open Access Statement: This is an Open Access article distributed in accordance with the Creative Commons Attribution-NonCommercial-NoDerivs 4.0 International License (CC BY-NC-ND 4.0), which permits the noncommercial replication and distribution of the article with the strict proviso that no changes or edits are made and the original work is properly cited (including links to both the formal publication through the relevant DOI and the license). See: https://creativecommons.org/licenses/by-nc-nd/4.0/.

\section{References}

1. Siegel RL, Miller KD, Jemal A. Cancer statistics, 2019. CA Cancer J Clin 2019;69:7-34.

2. Young JI, Mongoue-Tchokote S, Wieghard N, et al. Treatment and Survival of Small-bowel Adenocarcinoma in the United States: A Comparison with Colon Cancer. Dis Colon Rectum 2016;59:306-15.

3. Ryu DG, Choi CW, Kim SJ, et al. Possible indication of endoscopic resection in undifferentiated early gastric cancer. Sci Rep 2019;9:16869.

4. Kishino M, Nakamura S, Shiratori K. Clinical and Endoscopic Features of Undifferentiated Gastric Cancer in Patients with Severe Atrophic Gastritis. Intern Med 2016;55:857-62.

5. Wang X, Chen YH, Cai YQ, et al. Undifferentiated (spindle cell) pancreatic carcinoma: a case report with osteochondroid differentiation. JOP 2015;16:209-12.

6. Shah A, Khurana T, Freid L, et al. Undifferentiated Carcinoma with Osteoclast-Like Giant Cells of the Pancreas in a Patient with New Diagnosis of Follicular Non-Hodgkin's Lymphoma. ACG Case Rep J 2014;1:109-11.

7. Fujikawa T, Tanaka A, Abe T, et al. Undifferentiated carcinoma of the common bile duct with intraductal tumor thrombi: report of a case. Surg Today 2011;41:579-84.

8. Akatsu T, Ueda M, Shimazu M, et al. Primary undifferentiated spindle-cell carcinoma of the gallbladder presenting as a liver tumor. J Gastroenterol 2005;40:993-8.

9. Lim JH, Kim J, Kim SG, et al. Long-term clinical outcomes of endoscopic vs. surgical resection for early gastric cancer with undifferentiated histology. Surg Endosc 2019;33:3589-99.

10. Zhu ZL, Shi HP, Beeharry MK, et al. Expanding the indication of endoscopic submucosal dissection for undifferentiated early gastric cancer is safe or not? Asian J Surg 2020;43:526-31.

11. Park HJ, Jang KT, Choi DW, et al. Clinicopathologic analysis of undifferentiated carcinoma of the gallbladder. J Hepatobiliary Pancreat Sci 2014;21:58-63.

12. Yamada M, Furukawa D, Yazawa N, et al. Undifferentiated carcinoma of the ampulla of Vater. Surg Case Rep 2017;3:9.

13. Howlader N, Noone AM, Krapcho M, et al. editors. SEER Cancer Statistics Review, 1975-2017. Bethesda, MD: National Cancer Institute. Available online: https:// seer.cancer.gov/csr/1975_2017/, based on November 2019 SEER data submission, posted to the SEER web site, April 2020.

14. Kim HJ, Fay MP, Feuer EJ, et al. Permutation tests for joinpoint regression with applications to cancer rates. Stat Med 2000;19:335-51.

15. Rice TW, Chen LQ, Hofstetter WL, et al. Worldwide Esophageal Cancer Collaboration: pathologic staging data. Dis Esophagus 2016;29:724-33.

16. Uccella S, La Rosa S, Volante M, et al. Immunohistochemical Biomarkers of Gastrointestinal, Pancreatic, Pulmonary, and Thymic Neuroendocrine Neoplasms. Endocr Pathol 2018;29:150-68.

17. El Jabbour T, Lagana SM, Lee H. Update on hepatocellular carcinoma: Pathologists' review. World J Gastroenterol 2019;25:1653-65.

18. Singhi AD, Seethala RR, Nason K, et al. Undifferentiated carcinoma of the esophagus: a clinicopathological study of 16 cases. Hum Pathol 2015;46:366-75.

19. Park $\mathrm{CH}$, Kim EH, Kang JH, et al. Low Incidence of Synchronous or Metachronous Tumors after Endoscopic Submucosal Dissection for Early Gastric Cancer with Undifferentiated Histology. PLoS One 2016;11:e0147874.

20. Zhang L, Lee JM, Yoon JH, et al. Huge and recurrent undifferentiated carcinoma with osteoclast-like giant cells of the pancreas. Quant Imaging Med Surg 2018;8:457-60.

21. Brozek W, Kriwanek S, Bonner E, et al. Mutual 
associations between malignancy, age, gender, and subsite incidence of colorectal cancer. Anticancer Res 2009;29:3721-6.

22. Kakinoki R, Kushima R, Matsubara A, et al. Re-evaluation of histogenesis of gastric carcinomas: a comparative histopathological study between Helicobacter pylorinegative and H. pylori-positive cases. Dig Dis Sci 2009;54:614-20.

23. Kwon KJ, Shim KN, Song EM, et al. Clinicopathological characteristics and prognosis of signet ring cell carcinoma of the stomach. Gastric Cancer 2014;17:43-53.

24. Qiu J, Li X, He Y, et al. Distinct subgroup of the Ras family member 3 (DIRAS3) expression impairs metastasis and induces autophagy of gastric cancer cells in mice. J Cancer Res Clin Oncol 2018;144:1869-86.

25. Bryere J, Dejardin O, Launay L, et al. Socioeconomic status and site-specific cancer incidence, a Bayesian approach in a French Cancer Registries Network study. Eur J Cancer Prev 2018;27:391-8.

26. Gupta S, Tao L, Murphy JD, et al. Race/Ethnicity-, Socioeconomic Status-, and Anatomic Subsite-Specific Risks for Gastric Cancer. Gastroenterology 2019;156:59-62.e4.

27. Singh GK, Jemal A. Socioeconomic and Racial/Ethnic Disparities in Cancer Mortality, Incidence, and Survival in the United States, 1950-2014: Over Six Decades of Changing Patterns and Widening Inequalities. J Environ Public Health 2017;2017:2819372.

28. Savijärvi S, Seppä K, Malila N, et al. Trends of colorectal cancer incidence by education and socioeconomic status in Finland. Acta Oncol 2019;58:1557-63.

29. Jandova J, Ohlson E, Torres BSM, et al. Racial disparities and socioeconomic status in the incidence of colorectal cancer in Arizona. Am J Surg 2016;212:485-92.

30. Brooke HL, Talbäck M, Martling A, et al. Socioeconomic position and incidence of colorectal cancer in the Swedish

Cite this article as: Qiu J, Xin Y. Incidence and prognosis of undifferentiated cancers of the digestive system: a populationbased cohort study. Ann Transl Med 2021;9(1):15. doi: 10.21037/atm-20-1615 population. Cancer Epidemiol 2016;40:188-95.

31. Endo S, Nishikawa K, Yamada T, et al. Our experience of treating undifferentiated gastric carcinoma: report of four cases. Surg Today 2015;45:235-40.

32. Kohler BC, Goeppert B, Waldburger N, et al. An undifferentiated carcinoma at Klatskin-position with longterm complete remission after chemotherapy. Oncotarget 2018;9:22230-5.

33. Hiraki M, Kitahara K, Miyoshi A, et al. A long-term survivor of undifferentiated carcinoma of the liver successfully treated with surgical treatments: A case report and literature review. Int J Surg Case Rep 2018;51:45-9.

34. Reis Neto JA, Quilici FA, Cordeiro F, et al. Pre-operative radiotherapy in rectal cancer: evaluation of irradiation effects on cellular undifferentiation and its influence on prognosis. Hepatogastroenterology 1999;46:2825-30.

35. Kook MC. Risk Factors for Lymph Node Metastasis in Undifferentiated-Type Gastric Carcinoma. Clin Endosc 2019;52:15-20.

36. Sah SK, Li Y, Li Y. Undifferentiated carcinoma of the pancreas with osteoclast-like giant cells: a rare case report and review of the literature. Int J Clin Exp Pathol 2015;8:11785-91.

37. Muraki T, Reid MD, Basturk O, et al. Undifferentiated Carcinoma With Osteoclastic Giant Cells of the Pancreas: Clinicopathologic Analysis of 38 Cases Highlights a More Protracted Clinical Course Than Currently Appreciated. Am J Surg Pathol 2016;40:1203-16.

38. Moriguchi S, Kamakura T, Odaka T, et al. Clinical features of the differentiated and undifferentiated types of advanced gastric carcinoma: univariate and multivariate analyses. J Surg Oncol 1991;48:202-6.

39. Pyo JH, Lee H, Min YW, et al. Effect of age on the clinical outcomes of patients with early gastric cancer with undifferentiated-type histology. Surgery 2019;165:802-7. 